\title{
Reduced cerebral cortical but elevated striatal concentration of somatostatin-like immunoreactivity in dominantly inherited olivopontocerebellar atrophy
}

Stephen J Kish, Yves Robitaille, Munir El-Awar, Lawrence Schut, Linda DiStefano, Melvyn J Ball, Michael F Mazurek

\begin{abstract}
Somatostatin-like immunoreactivity (SLI) was measured in the brains of nine patients with dominantly inherited olivopontocerebellar atrophy (OPCA), who all had a marked deficit of the cholinergic marker cholineacetyltransferase (ChAT) in the cerebral cortex and striatum. Mean concentrations of SLI in OPCA were significantly reduced by $42-58 \%$ in parietal and occipital cortices and frontal cortical eye fields, but were normal in other cortical areas, including two subdivisions of the temporal cortex which show marked depletions of both SLI and ChAT in Alzheimer's disease. This dissociation of SLI and ChAT indicates that a cortical cholinergic deficit does not invariably lead to reduction of somatostatin. In the caudate nucleus, the region of OPCA brain having the most severe ChAT deficit ( $-81 \%)$, SLI levels were significantly elevated by $46 \%$ and were negatively and significantly correlated with ChAT activities $(r=-0.66)$. The SLI alterations could be due to abnormal somatostatin metabolism or release, or an increased number of somatostatin-containing neurons and could contribute to the brain dysfunction of OPCA.
\end{abstract}

(F Neurol Neurosurg Psychiatry 1993;56:1013-1015)

Reduced concentration of somatostatin-like immunoreactivity (SLI) has been reported in the cerebral cortex of patients with several progressive neurodegenerative conditions including Alzheimer's disease, ${ }^{1-4}$ Parkinson's disease with dementia ${ }^{5}$ (but see Leake et al $^{3}$ and Whitford et $a l^{6}$ ), and senile dementia of the Lewy body type. ${ }^{3}$ The experimental observation that this neuropeptide may serve a neurotransmitter or neuromodulatory role (for review see Beal et $\mathrm{al}^{7}$ ) implicates somatostatin in the pathophysiology of the above conditions. Degeneration of cholinergic neurons innervating the cerebral cortex, as shown by reduced levels of cholineacetyltransferase (ChAT), is also associated with each of these (dementing) disorders. ${ }^{135}$ This, together with the observations of a significant correlation in several brain areas between the reductions of brain SLI and ChAT levels (cf. Epelbaum et $a l^{5}$ ), suggest that a primary degeneration of one of the two neuronal types might produce secondary (trans-synaptic) changes in the other. Arguing against this notion, however, are the published reports demonstrating dissociation between cerebral cortical SLI and ChAT changes in several rare human brain disorders (focal Alzheimer's disease, ${ }^{8}$ "dysphasic dementia"'), together with the experimental observation of a lack of influence of (acute) cholinergic neuron damage on cerebral cortical SLI concentration. ${ }^{10}$ In order to further clarify the nature of the relationship between brain somatostatin and cholinergic abnormalities in the human we have examined the behaviour of cerebral cortical and striatal (caudate and putamen) SLI, together with ChAT, in necropsied brains of nine patients with dominantly inherited olivopontocerebellar atrophy (OPCA), a cerebellar ataxia disorder in which affected members of several different pedigrees have a marked cerebral cortical and striatal cholinergic (ChAT) reduction. ${ }^{1112}$

\section{Patients and methods}

Necropsied brain was obtained from nine patients with OPCA and 10 control subjects dying without evidence of neurological or psychiatric disease who were matched with respect to age (OPCA mean 39 (SE 3); controls: 40 mean (SE 5) years; $p>0.05$; Student's two tailed $t$ test) and postmortem time (OPCA: 10 (2) hours; controls: 11 (2) hours; $p>0.05$ ). At necropsy one half-brain was frozen at $-80^{\circ} \mathrm{C}$ for biochemical investigation whereas the other half-brain was fixed in formalin for neuropathological analysis (YR, MB). The nine OPCA patients were from pedigrees $S(n=6)$, Se $(n=1), C(n=$ $1)$, and $D(n=1)$ in which at least three successive generations have been affected. We have previously reported a cerebral cortical/ striatal ChAT deficit in the brains of five of the six pedigree $S$ patients examined in the present study. ${ }^{12}$ All patients had severe limb 
ataxia and dysarthria and were either wheelchair-bound or bedridden. Formal neuropsychological testing had been conducted on three of the pedigree $S$ patients. ${ }^{12}$ Neurological assessment of the other three pedigree $S$ patients, and the two patients from pedigrees Se and C, did not reveal any clinically significant dementia. Moderate to severe dementia was observed only in the single patient from pedigree $D$, in which neuropathological analysis revealed moderate cerebral cortical neuronal loss (see below). Neuropathological analysis confirmed the diagnosis of OPCA with severe Purkinje cell loss in cerebellar cortex, and neuronal loss with gliosis in the pons and inferior olives. Microscopic analysis of the cerebral cortex (frontal, parietal, temporal, occipital) showed no evidence of neuronal cell loss or gliosis in six of the nine patients (pedigree $S, n=4$; pedigree $S e, n=1$; pedigree $C, n=1$ ) with two patients (pedigree $S$ ) showing slight neuronal cell loss and gliosis in parietal cortex (other cerebral cortical areas within normal limits). The single patient from pedigree D showed moderate cerebral cortical neuronal loss with little gliosis affecting primarily the deeper cortical layers. The neuronal population of the striatum (caudate and putamen) was within normal limits in six of the nine patients whereas in three patients (pedigree $S$, $\mathrm{n}=2$; pedigree $\mathrm{C}, \mathrm{n}=1$ ) $\mathrm{minimal} / \mathrm{mild}$ neuronal cell loss could be observed; these changes were not associated with specific abnormalities on silver stains. No senile plaques or neurofibrillary tangles were observed in the brains of any of the OPCA patients. SLI was measured by radioimmunoassay as previously described. ${ }^{2}$ The assay recognises amino acids 6 to 10 of the tetradecapeptide somatostatin. Somatostatin 14 and 28 are recognised on an equimolar basis. ChAT activity was determined using the radiochemical procedure of Fonnum. ${ }^{13}$

\section{Results}

Compared with the controls, mean levels of SLI were significantly reduced $(p<0.05$ or less, Student's two tailed $t$ test) by $42-58 \%$ in Brodmann areas 8 (frontal eye field), $7 \mathrm{~b}$ (parietal cortex), and 18 (occipital cortex) but were normal in areas 6 (pre-motor cortex), 20

Table Somatostatin-like immunoreactivity (SLI) and choline acetyltransferase (ChAT) in necropsied human brain: control vs dominantly inherited olivopontocerebellar atrophy (OPCA)

\begin{tabular}{|c|c|c|c|c|}
\hline \multirow[b]{2}{*}{ Brain area } & \multicolumn{2}{|l|}{$S L I$} & \multicolumn{2}{|l|}{$C h A T$} \\
\hline & Control & $O P C A$ & Control & $O P C A$ \\
\hline $\begin{array}{l}4 \\
6 \\
7 \mathrm{~b} \\
8 \\
18 \\
20 \\
38 \\
\text { Caudate } \\
\text { Putamen }\end{array}$ & $\begin{array}{ll}28.9 & (3 \cdot 6) \\
25 \cdot 5 & (2 \cdot 9) \\
28 \cdot 8 & (2 \cdot 1) \\
31 \cdot 1 & (2 \cdot 0) \\
25.7 & (2 \cdot 0) \\
31.4 & (3 \cdot 9) \\
34.9 & (5 \cdot 3) \\
34 \cdot 4 & (4 \cdot 4) \\
44.2 & (7 \cdot 2)\end{array}$ & $\begin{array}{l}19.7(2.8) \\
21.6(1.9) \\
12.0(1.9)^{\star \star \star} \\
18 \cdot 1(3.3)^{\star \star} \\
14.5(2 \cdot 1)^{\star \star} \\
35.3(6.6) \\
39.0(5 \cdot 5) \\
50.2(5.2)^{\star} \\
60.1(7.9)\end{array}$ & $\begin{array}{cc}1.90 & (0.16) \\
1.69 & (0.18) \\
1.23 & (0.10) \\
1.22 & (0.10) \\
0.92 & (0.09) \\
1.28 & (0.11) \\
1.47 & (0.15) \\
40.2 & (3.5) \\
51.1 & (5.2)\end{array}$ & $\begin{array}{r}0.65(0.08)^{\star \star \star \star} \\
0.55(0.05)^{\star \star \star} \\
0.44(0.06)^{\star \star \star} \\
0.50(0.06)^{\star \star \star} \\
0.50 \\
0.24(0.02)^{\star \star \star} \\
0.46(0.06)^{\star \star \star} \\
0.73(0.06)^{\star \star \star} \\
7.6 \\
22.6(1.6)^{\star \star \star \star} \\
22.7(2.8)^{\star \star \star \star}\end{array}$ \\
\hline
\end{tabular}

Values (SLI, pg somatostatin-14/mg tissue; ChAT, pmol $\mu g$ protein/10 minutes) represent mean (SE) of nine patients with OPCA and 10 control subjects. Numbered brain areas are

${ }^{\star} \mathrm{p}<0.05,{ }^{\star \star} \mathrm{p}<0.01,{ }^{\star \star \star} \mathrm{p}<0.001$; Student's two-tailed $t$ test. (inferior temporal gyrus), and 38 (temporal pole), and in the putamen (see table). The $32 \%$ mean reduction of SLI in area 4 (precentral gyrus) just missed statistical significance at the 0.05 level. SLI concentration, on average, was significantly elevated by $46 \%$ (p $<0.05$ ) in the caudate nucleus of the OPCA patients. By comparison, mean ChAT activity was markedly and significantly $(p<0.001)$ reduced by 50 to $81 \%$ in all nine examined brain areas with the greatest reduction occurring in the caudate nucleus. In the OPCA group no statistically significant $(p>0.05)$ correlation was observed between SLI and ChAT levels in any of the seven cerebral cortical areas or in the putamen. In the caudate nucleus, however, a significant $(p<0.05)$ negative correlation $(r=-0.66)$ was observed between SLI and ChAT values in the OPCA patients.

\section{Discussion}

Whereas the brain neuropathology of affected patients from the examined OPCA families has been generally assumed to be restricted primarily to the cerebellum and lower brain stem, our data show abnormal levels of SLI in OPCA cerebral cortex and striatum with a regional pattern differing from that reported for other brain neurodegenerative conditions. Our finding of decreased SLI in OPCA cerebral cortex can be compared with Alzheimer's disease, in which a cortical SLI deficit is a feature of this condition, but in which, unlike OPCA, SLI levels are reduced by $42-58 \%$ in the temporal lobe $^{4}$ and are normal in striatum. ${ }^{14}$ Increased striatal SLI, observed in our OPCA patients, is also found in Huntington's disease, ${ }^{15}$ but in the latter condition none of the (24) cerebral cortical areas examined showed the decreased SLI we have observed in OPCA.

In cerebral cortex somatostatin-immunoreactive neurons are found in a subpopulation of locally projecting neurons (cf Beal et $a l^{7}$ ). The SLI deficit in OPCA cerebral cortex could be explained by loss of somatostatincontaining neurons. However, our preliminary observation ${ }^{16}$ that the SLI deficient areas of OPCA cerebral cortex contain normal concentration of neuropeptide $\mathrm{Y}$ (which, to a large extent is co-localised with somatostatin in human cerebral cortical neurons), ${ }^{17}$ argues against neuronal cell loss as an explanation for the reduced SLI and points towards a change in somatostatin release, metabolism, or prosomatostatin metabolism. This differs from Alzheimer's disease cerebral cortex in which reduced levels of both SLI (see above) and neuropeptide $\mathrm{Y}^{18}$ are observed.

Unlike the cerebral cortex, mean SLI concentration in striatum (caudate) was actually elevated in the OPCA patients. Increased striatal levels of SLI are also observed in Huntington's disease, ${ }^{15}$ which is associated with marked atrophy of the striatum. Since none of our OPCA patients had, upon gross inspection, striatal atrophy, the SLI elevation is unlikely to be consequent to any artefactural increase resulting from tissue shrinkage. 
The regional patterns of the SLI and ChAT changes were dissimilar, the most striking difference being the temporal cortex, which consistently shows both ChAT and SLI loss in Alzheimer's disease, ${ }^{1-4}$ but which, in OPCA, shows only a cholinergic reduction. Our data indicate therefore that a loss of cholinergic innervation to cerebral cortex does not inevitably result in a cortical somatostatin deficit. SLI levels were elevated in the caudate nucleus, the region of OPCA brain found to have the most marked ChAT reduction. In striatum, somatostatin immunoreactivity is contained in medium sized aspiny neurons where it is co-localised with neuropeptide $\mathrm{Y}$ and NADPH diaphorase. This neuronal population is distinct from the large aspiny cholinergic interneuron of the striatum..$^{7}$ Observation of a significant negative correlation between caudate SLI and ChAT levels suggests the interesting possibility that a primary loss or downregulation of striatal cholinergic neurons might result in nerve terminal sprouting or upregulated neuropeptide synthesis in the striatal somatostatinergic neurons.

The functional significance of the brain somatostatin changes in OPCA, as well is in Alzheimer's disease, is uncertain. In Alzheimer's disease SLI has been observed in neuritic plaques ${ }^{19}$ and in neurons containing neurofibrillary tangles, ${ }^{20}$ suggesting that this neuropeptide could play a role in the neurodegenerative process. However, senile plaques and neurofibrillary tangles were not observed in the brain of any of our (young, mean age 39 years) OPCA patients, indicating that formation of these brain pathological markers of neurodegeneration cannot be an invariable consequence of cortical somatostatin loss. The observations of a correlation between brain somatostatin levels in Alzheimer's disease ${ }^{3}$ and Parkinson's disease with dementia ${ }^{5}$ with a degree of cognitive impairment has also implied a role for somatostatin in cognition. However, in our study clinically significant dementia was observed only in the single patient from pedigree $\mathrm{D}$, with pedigree $\mathrm{S}$ patients typically having only clinically mild frontal subcortical type cognitive deficits upon formal neuropsychological testing. ${ }^{1221}$

This, suggests that a cerebral cortical somatostatin deficit of the magnitude and regional pattern observed in OPCA is not sufficient to produce a clinically disabling dementia, but could provide the basis for part of the mild cognitive dysfunction in this disorder. The experimental finding of increased striatal dopamine release in the rodent following local somatostatin administration ${ }^{22}$ suggests that somatostatin might play an important role in the control of dopamine release at nigrostriatal neuronal terminals; this observation may be related to our previous demonstration of increased dopamine turnover (elevated dopamine metabolite/ neurotransmitter ratio) in striatum of OPCA patients. $^{23} \mathrm{We}$ conclude that abnormal somatostatin function in both cerebral cortex and striatum, as suggested by our data, could contribute to part of the brain dysfunction of OPCA.

This study was supported by the US NIH (NINDS no 26034 to SK) and the Ontario Mental Health Foundation and Alzheimer Association of America (to MM). Drs Kish and Mazurek are career scientists of the Ontario Ministry of Health.

Presented in part at the 117th annual meeting of the American Neurological Association, October, 1992, Toronto, Canada.

1 Rossor MN, Emson PC, Mountioy CQ, Roth M, Iversen LI. Reduced amounts of immunoreactive somatostatin LL. Reduced amounts of immunoreactive somatostatin in the temporal cortex in senile

2 Beal MF, Mazurek MF, Svendsen CN, Bird ED, Martin JB. Widespread reduction of somatostatin-like immunoreactivity in the cerebral cortex in Alzheimer's disease. Ann Neurol 1986;20:489-95.

3 Leake A, Perry EK, Perry RH, et al. Neocortical concentrations of neuropeptides in senile dementia of the Alzheimer and Lewy Body type: comparison with Parkinson's disease and severity correlations. Biol Psychiatry 1991;29:357-64.

4 Mazurek MF, Beal MF. Cholecystokinin and somatostatin in Alzheimer's disease postmortem cerebral cortex. Neurology 1991;41:716-9.

5 Epelbaum J, Ruberg M, Moyse E, Javoy-Agid F, Dubois B, Agid Y. Somatostatin and dementia in Parkinson's B, Agid Y. Somatostatin and dem

6 Whitford C, Candy J, Edwardson J, Perry R. Cortical somatostatinergic system not affected in Alzheimer's and Parkinson's disease. $\mathcal{F}$ Neurol Sci 1988;86:13-8.

7 Beal MF, Uhl G, Mazurek MF, Kowell N, Martin JB Somatostatin: alterations in the central nervous system in neurological diseases. In: Martin JB, Barchas JD, eds. Neuropeptides in neurologic and psychiatric disease. New York: Raven Press, 1986:215-57.

8 Jagust WJ, Davies P, Tiller-Borcich JK, Reed BR. Focal Alzheimer's disease. Neurology 1990;40:14-9.

9 Mehler MF, Horoupian DS, Davies P, Dickson DW. Reduced somatostatin-like immunoreactivity in cerebral cortex in nonfamilial dysphasic dementia. Neurology 1987;37:1 148-453.

10 Fine A, Pittaway K, de Quidt M, Czudek C, Reynolds GP. Maintenance of cortical somatostatin and monoamine levels in the rat does not require intact cholinergic innervation. Brain Res 1987;406:326-9.

11 Kish SJ, Currier RD, Schut L, Perry TL, Morito CL Brain choline acetyltransferase reduction in dominantly inherited olivopontocerebellar atrophy. Ann Neurol 1987;22:272-5

12 Kish SJ, Robitaille Y, El-Awar M, et al. Non-Alzheimertype pattern of brain cholineacetyltransferase reduction in dominantly inherited olivopontocerebellar atrophy. Ann Neurol 1989;26:362-7.

13 Fonnum F. A rapid radiochemical method for the determination of choline acetyltransferase. $\mathcal{F}$ Neurochem 1975 ; 24:407-9.

14 Beal MF, Mazurek MF, McKee MA. The regional distribution of somatostatin and neuropeptide $\mathrm{Y}$ in control and Alzheimer's disease striatum. Neurosci Lett 1987;79: 201-6.

15 Beal MF, Mazurek MF, Ellison DW, et al. Somatostatin and neuropeptide $\mathrm{Y}$ concentrations in pathologically graded cases of Huntington's disease. Ann Neurol 1988; 23:562-9.

16 Mazurek MF, Kish SJ. Neuropeptide abnormalities in cerebral cortex of olivopontocerebellar atrophy (OPCA) postmortem brain. Ann Neurol 1992;32:271-2

17 Chronwall BM, Chase TN, O'Donohue TL. Coexistence of neuropeptide $\mathrm{Y}$ and somatostatin in rat and human cortical and rat hypothalamic neurons. Neurosci Lett 1984;52:213-7.

18 Beal MF, Mazurek MF, Chattha GK, Svendsen CN, Bird ED, Martin JB. Neuropeptide Y immunoreactivity is ED, Martin JB. Neuropeptide Y immunoreactivity is reduced in cerebral co

19 Armstrong DM, Leroy S, Shields D, Terry RD. Somatostatin-like immunoreactivity within neuritic plaques. Brain Res 1985;338:71-9.

20 Roberts GW, Crow TJ, Polak JM. Location of neuronal tangles in somatostatin neurones in Alzheimer's disease. Nature 1985;314:92-4.

21 Kish SJ, El-Awar M, Schut L, Leach L, Oscar-Berman M, Freedman M. Cognitive deficits in olivopontocerebellar atrophy: implications for the cholinergic hypothesis of Alzheimer's dementia. Ann Neurol 1988;24:200-6.

22 Chesselet MF, Reisine TD. Somatostatin regulates dopamine release in rat striatal slices and cat caudate nuclei. 7 Neurosci 1983;3:232-6.

23 Kish SJ, Robitaille Y, El-Awar M, et al. Striatal monoamine neurotransmitters and metabolites in dominantly inherited olivopontocerebellar atrophy. Neurology 1992; 42:1573-7. 